\title{
Liver histology and follow up of 68 patients with ulcerative colitis and normal liver function tests
}

\author{
U Broomé , H Glaumann, R Hultcrantz
}

\begin{abstract}
Hepatobiliary disorders are well known complications in patients with ulcerative colitis but it is not possible to predict those patients with ulcerative colitis who will eventually devleop liver disease. In this study, liver biopsies from 74 patients with ulcerative colitis have been reevaluated. None of the patients showed clinical or biochemical signs of liver disease at the time of biopsy. Thirty seven $(50 \%)$ had a completely normal liver biopsy. The others showed minimal portal inflammation or fatty infiltration. The biopsies of three patients displayed concentric, periductular fibrosis, or so called 'onion skin' lesions. None showed other signs of fibrosis or cirrhosis. The histological findings were unrelated to either activity or extent of colitis, except for the onion skin lesions which were seen exclusively in biopsies of patients with involvement of the total colon. Sixty eight of the 74 patients were reviewed after a mean interval of 18 years. The majority had no symptoms of hepatobiliary disorders and only two had developed liver disease; one suffered from cryptogenic cirrhosis, possibly due to non-A, non-B hepatitis and the other of an autoimmune liver disease and later developed a bile duct carcinoma. Both were women with total colonic involvement. At the time of the first liver biopsy one showed no histological abnormalities and the other only minor fatty infiltration. Thus, minor abnormalities in liver tissue are common in patients with ulcerative colitis without biochemical evidence of liver disease. The morphological changes are of little help in predicting the future risk of a patient with ulcerative colitis developing a clinically relevant hepatobiliary complication. The absence of biochemical parameters for liver disease during the early years of ulcerative colitis predict a favourable longterm diagnosis as regards hepatobiliary complications.
\end{abstract}

Departments of Medicine U Broomé R Hultcrantz

Infectious Diseases, and Pathology, Huddinge University Hospital and Roslagstull Hospital, Stockholm, Sweden H Glaumann

Correspondence to: Ulrika Broomé, MD, Huddinge Broomé, MD, Huddinge University Hospital,
Huddinge, Sweden.

Accepted for publication 10 July 1989
In 1874 Thomas' described an association between ulcerative colitis and liver disease. Since then, many reports have confirmed this finding ${ }^{23}$ and it is today considered a fact that the incidence of liver disease in patients with ulcerative colitis is greater than in the general population. ${ }^{3-5}$ The most common histological findings are fatty infiltration and portal inflammatory cell infiltrates consisting of collections of lymphocytes and plasma cells. Often, but not always, these cells are concentrated around the bile ducts, and eventually hyaline fibrous tissue surrounding bile ducts may develop. ${ }^{2-7}$ There have been attempts to correlate the histological changes seen in liver tissue to the severity and extent of the colitis ${ }^{48}$ but so far no such correlation has been found.

In 1966, primary sclerosing cholangitis was recognised in patients with ulcerative colitis. ${ }^{910}$ Initially, it was described as an unusual finding, ${ }^{8-}$ ${ }^{10}$ but with an increased awareness of the disease and with the availability of endoscopic retrograde cholangiography in addition to a possible absolute increase of the disease, primary sclerosing cholangitis is today found in $2-4 \%$ of all patients with ulcerative colitis. ${ }^{11}{ }^{12}$ Except for one study by Perrett et al, ${ }^{13}$ who found minor histological abnormalities in six of 13 patients with ulcerative colitis without clinical or biochemical evidence of liver disease, little is known about the incidence of alterations in the liver in patients with ulcerative colitis in general. Also, aetiological factors leading to liver disease in patients with ulcerative colitis are not clarified.

In the present study liver biopsies from 74 patients suffering from ulcerative colitis with normal liver function tests have been reexamined according to the new classification system of liver biopsies. ${ }^{14}$ The patients were followed up in order to assess if patients with ulcerative colitis, but lacking biochemical parameters of liver disease, run a lower risk of developing hepatobiliary complications compared with all patients with ulcerative colitis. We also wanted to establish if any specific histological abnormality would be of value in predicting the future risk that a patient with ulcerative colitis will develop liver disease.

\section{Methods}

\section{PATIENTS}

Between 1958 and 1968, at St Erik's Hospital in Stockholm, Sweden, liver biopsies as well as biochemical liver function tests were routinely performed on patients with ulcerative colitis. In 78 of the patients, the liver function was more extensively examined and was found to be completely normal. Four of the patients later developed Crohn's disease and were therefore excluded from our present study.

Clinical data were obtained from the hospital records at the time of the biopsy. There were 34 men and 40 women. The mean age at onset of ulcerative colitis was 31 years $(8-69)$. At this time the Ethics committee system was not operating in Sweden but as far as we know informed consent was not obtained. The diagnosis of ulcerative colitis was based on the following criteria: a history of diarrhoea and/or rectal bleeding for six weeks or more; at least one sigmoidoscopy showing friability of the mucosa with contact bleeding and/or petechial haemorrhages and/or ulceration with intervening inflam- 
mation of the mucosa, and a barium enema with radiological evidence of ulceration or narrowing or shortening of the colon; and characteristic microscopical changes in the biopsy. ${ }^{15}$

The extent of the colitis was determined by sigmoidoscopy and barium enema. Thirty seven patients were found to have total involvement of the colon, 28 left sided involvement and nine had proctitis. The inflammatory activity of the colitis had been determined. Remission was defined as a state in which the patient had no bowel symptoms and the sigmoidoscopy showed no activity. The colitis was defined as active when the sigmoidoscopy showed oedema, excess mucus, signs of bleeding and/or ulceration and the patient had symptoms of diarrhoea. Fifteen patients were in remission, and in 59 patients the colitis was active. Two patients had been subjected to cholecystectomy, and one had previously suffered from hepatitis $A$. Three patients were classified as over consumers of alcohol while the others consumed no alcohol or only small amounts at weekends. Sulphasalazine was taken by 21 of the patients, corticosteroids by 17 and local corticosteroids by 27 patients. Guanicil, a non-absorbable sulphonamide, was taken by nine patients and 10 patients were taking no medicine at the time of the liver biopsy. Thus, because of the various medical treatments and the small group of patients, it was not meaningful to correlate the histological findings to the drugs taken.

The liver function in all patients was evaluated by bilirubin, transaminases, alkaline phosphatase, and Bromsulphalein retention tests. All these tests were normal in all 74 patients. Liver biopsy was performed on average seven years after the onset of the ulcerative colitis. The percutaneous technique was used and each biopsy stained with haematoxylin-eosin and van Gieson. The biopsies were reevaluated by one liver pathologist and one hepatologist according to the classification system of liver diseases ${ }^{14}$ and without knowing the patients identity. Special emphasis was placed on alterations in the portal tracts such as inflammation and fibrosis. Hepatocellular degeneration and fatty infiltration were also carefully assessed. Portal inflammation and fatty infiltration were subjectively quantified on an arbitary scale from 0 to ++++ .

Through The National Population Registry it was possible to trace 68 patients. The time of follow up was taken for 60 patients as the last recorded hospital visit when a clinical examination was carried out with measurements of transaminases, alkaline phosphatase and bilirubin. Biochemical data of the liver function could not be found for two patients and the last recorded clinical examination was then taken as the time of follow up. Four patients had died and

TABLE Survey of follow up of 74 patients with ulcerative colitis

\begin{tabular}{lc}
\hline Type of investigation & Patients $(n)$ \\
\hline Physical examination and liver function tests & 60 \\
Physical examination & 2 \\
Autopsy & 4 \\
Liver biopsy, physical examination and liver & 2 \\
function tests & 6 \\
Lost at follow up & \\
\hline
\end{tabular}

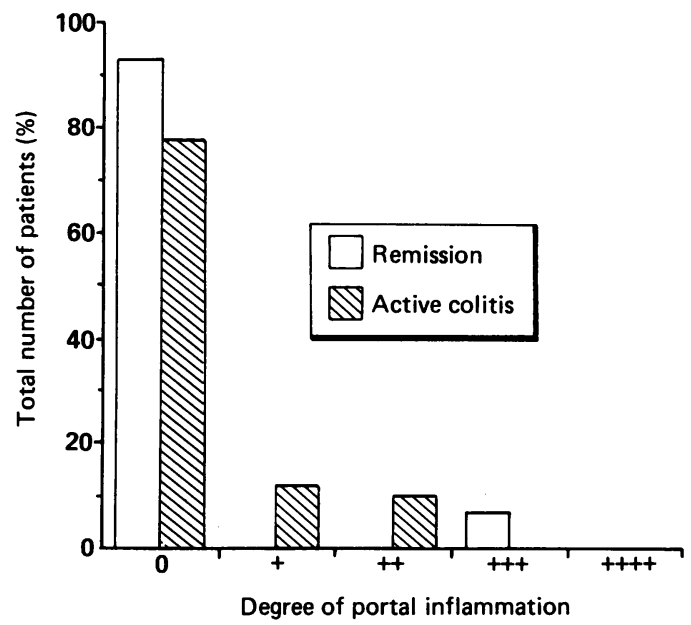

Figure 1: Comparison between the degree of portal inflammation in patients with active colitis and with colitis in remission.

all had been autopsied. The time of death was then taken as the time of follow up. Among the six patients whom it was not possible to follow up, we know that two women died at the age of 92 and 80 respectively. The fate of the remaining four patients are unknown to us today (Table). The mean time of follow up was 18 years $(0-28)$.

\section{Results}

\section{LIVER BIOPSY INTERPRETATION}

Among the 74 patients studied, $37(50 \%)$ had a completely normal initial liver biopsy. Twenty three biopsies showed fatty infiltration, nine portal inflammation, and in five there were both fatty infiltration and portal inflammation. Three displayed fatty infiltration sufficient to make the diagnosis of fatty liver (more than $50 \%$ of the hepatocytes involved). One of these three patients was in clinical remission, whereas two suffered from active colitis. In the liver biopsy from one patient in remission more extensive portal inflammation was seen.

The quantitative data regarding portal inflammation and lobular changes in relation to the inflammatory activity of the colitis are given in Figures 1 and 2, respectively, where it can be seen that there was no correlation between the

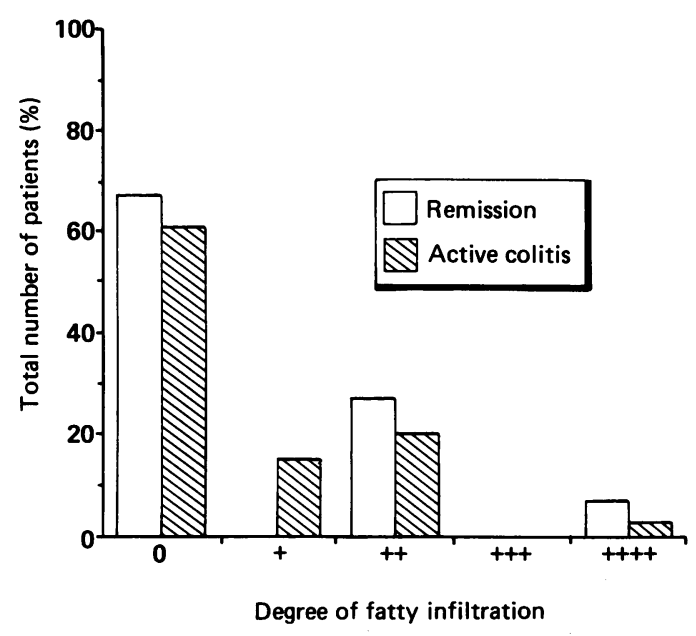

Figure 2: Comparison between the degree of fatty infiltration in patients with active colitis and with colitis in remission. 
Figure 3: Degree of portal inflammation in relation to the extent of involvement of the colon.

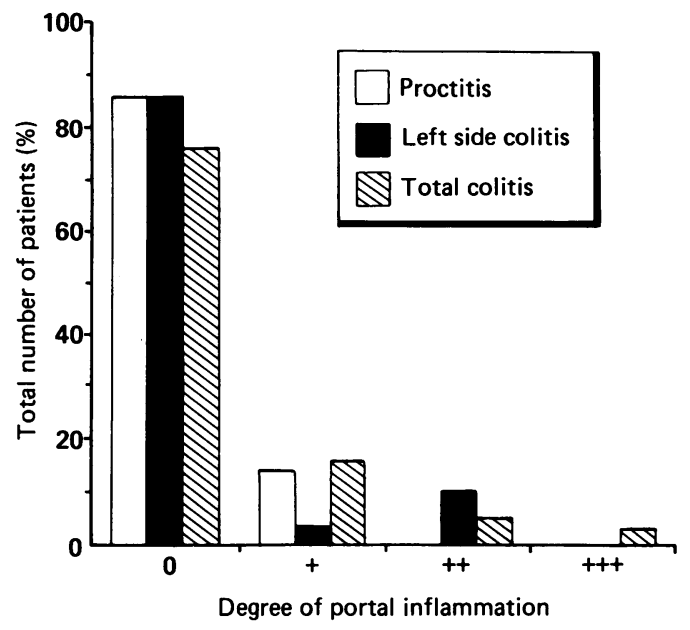

histological findings in the liver and the activity of the colitis defined as remission or active. Furthermore, only one patient had more than slight portal inflammation, the rest had very minor or no inflammation. Fatty infiltration was sparse and evidently not correlated to the portal inflammatory activity.

The extent of the involvement of the colitis was correlated to the histological findings, namely portal inflammation (Fig 3) and fatty infiltration (Fig 4). There was, however, no clear cut correlation between these two histological abnormalities and the extent of the colitis. Fibrosis was only seen in three patients. In the biopsies of these patients the interlobular bile ducts were surrounded by concentric fibrosis, or 'onion-skin' lesions, previously described in patients with sclerosing cholangitis. These patients who all displayed total colonic involvement are described below.

\section{PATIENT I}

A man born in 1926. The onset of ulcerative colitis was in 1949 and involved the total colon. A liver biopsy was performed in 1965, while the patient was in remission showing two portal tracts with concentric periductular fibrosis but no signs of inflammatory activity around the bile ducts or elsewhere in the portal tracts. Fatty infiltration was graded to + . The patient was otherwise healthy and did not abuse alcohol. No treatment for ulcerative colitis or any other disease was given at the time of the biopsy.

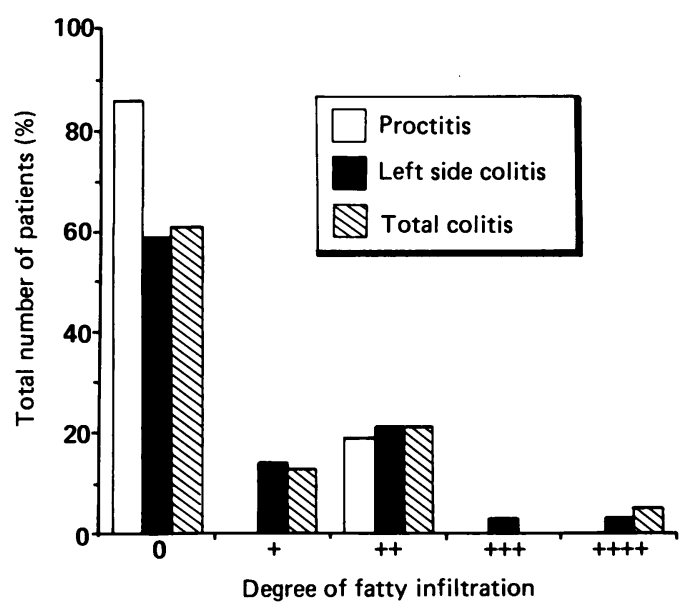

Twenty three years after the biopsy the patient showed no clinical or biochemical signs of liver disease.

PATIENT 2

A woman born in 1927. Onset of ulcerative colitis was in 1949 involving the total colon. Liver biopsy was performed in 1967, at which time the colitis was in remission. The patient was treated for many years with sulphasalazine. There was no other ailment. The biopsy showed portal tracts with typical fibrosis around the bile ducts but no inflammatory infiltrates around these. In some other portal tracts, however, there were signs of chronic inflammation with small lymphoid aggregates. In 1987, 20 years after the biopsy, the patient was free from any clinical or biochemical parameters of liver disease.

\section{PATIENT}

A man born in 1949 with total ulcerative colitis starting in 1957. A liver biopsy was performed in 1967 when the colitis was active. He was treated with sulphasalazine and corticosteroids at the time of the biopsy. He had no history of excessive alcohol ingestion. The liver biopsy showed concentric periductular fibrosis without any signs of inflammation. As in patient 2 , however, there was inflammation graded to ++ in some portal tracts and fatty infiltration graded to + . Thirteen years later the patient showed no clinical or biochemical evidence of liver disease.

\section{FOLLOW UP}

Of the 68 patients traced two had developed evidence of liver abnormalities at the time of follow up. The first was a woman aged 52 years with onset of ulcerative colitis at an age of 27 . The entire colon was involved. Salazopyrin was taken for many years. The first liver biopsy was performed in 1965 during a fulminant attack of the colitis, two years after the onset of the ulcerative colitis. The biopsy showed no histological abnormalities. In 1967 a colectomy was performed because of chronic persistent colitis. The liver was normal at the time of surgery. In the years before colectomy she received several units of blood during severe attacks of ulcerative colitis with concomitant anaemia and in 1970 the patient was hospitalised because of jaundice. The aetiology of the jaundice has not been able to be established. In 1973 the transaminases were slightly raised and there was a third liver biopsy which on reexamination showed sparse chronic inflammation and some piecemeal necrosis in the portal tracts. A radionucleotide scan showed a normal sized liver but an enlarged spleen. Smooth muscle antibodies, mitochondrial antibodies and antinuclear factor were negative and the patient has no symptoms or signs of liver disease. The transaminases remained slightly raised but less than twice the upper normal level, the alkaline phosphatase and bilirubin values were normal. The prothrombin time was somewhat prolonged. A new biopsy in 1985, 12 years later, showed cirrhosis with moderate chronic 
inflammation in the portal tracts and piecemeal necrosis, but no lobular changes and no bile duct proliferation. An endoscopic retrograde cholangiography has not yet been performed. The second patient to develop liver disease is a woman, born in 1931. Ulcerative colitis started in 1949 with total colonic involvement and a liver biopsy was performed in 1959 when the colitis was active, showing minor fatty infiltration but no portal inflammation. She had been treated with salazopyrin for many years. There was no history of alcohol abuse. In 1971 the patient became jaundiced and tired, and lost weight. The transaminases were raised maximally, nine times the upper normal concentration whereas the alkaline phosphatases activity never reached more than slightly raised levels. The antinuclear factor was positive in dilution $1 / 1600$ and antibodies against smooth muscle positive 1/25. Hepatitis B surface antigen was negative. A liver biopsy showed bile duct proliferation, periportal inflammation and a portal cirrhosis was suspected. Chronic active hepatitis was then considered possible and corticosteroids were given. Clinical and biochemical improvement occurred with a decrease in the transaminases to twice the upper normal level and normalisation of the alkaline phosphatase. The antinuclear factor became negative. The corticosteroid treatment was gradually withdrawn. A new episode with jaundice and fatigue occurred in 1976. The transaminases were again high. Antibodies against smooth muscle were positive in $1 / 100$. The patient was again successfully treated with corticosteroids, and the biochemical and clinical signs of liver disease slowly disappeared. In 1977, a repeat liver biopsy was performed, showing some fatty infiltration and some slightly enlarged portal tracts, but no signs of cirrhosis or chronic hepatitis. From 1978 to 1985 transaminases and alkaline phosphatase were essentially within the normal limits. Prothrombin time and albumin were normal. A cholecystectomy was performed in 1982 and in 1987 an ileorectal anastomosis was carried out for a benign stenosis between the descending and sigmoid colon.

In early 1988 the transaminases again increased to maximally 10 and the alkaline phosphatase to five times the upper normal limit. A computed tomography of the liver was performed revealing an enlarged liver with the intrahepatic bile ducts being irregular and dilated. A new liver biopsy showed moderate fatty infiltration, fibrotic bridging, general bile duct proliferation, and malignant transformation of some bile ducts. No endoscopic retrograde cholangiography has been performed.

\section{Discussion}

This study of a comparatively large group of patients suffering from ulcerative colitis initially without any clinical or biochemical evidence of liver disease shows that minor histological changes are common in liver biopsies from these patients. According to De Dombal et $a l^{4}$ and Eade, ${ }^{8}$ it should be possible to relate histological alterations such as fatty infiltration and portal inflammation to the clinical course of the colitis.
In our study, no such correlation was found between the activity and the extent of the involvement of the large intestine on one hand and the histological changes in the liver on the other. In $4 \%$ of our patients, periductal concentric fibrosis or 'onion skin lesions' were found These lesions were exclusively observed in patients with total colonic involvement, but otherwise without relation to the inflammatory activity of the colon.

In a study of patients with ulcerative colitis, who had been subjected to liver biopsy because of biochemical evidence of liver disease, the biopsies disclosed a high frequency of histological alterations, often coinciding with findings indicating primary sclerosing cholangitis. ${ }^{16}$ In this study many patients had biopsy findings indicating primary sclerosing cholangitis - for example, periductal fibrosis and inflammation in the portal zones. This group of patients apparently constituted a different population from ours, they had clinical or biochemical signs of liver disease, and consequently, also had a much poorer prognosis than our patients. One third of the patients had died 10 to 15 years after the biopsy compared with our study in which only two patients (3\%) developed clinically significant liver disease. We have no evidence that any of our patients died of liver disease although the exact fate of four of the patients is unknown.

Primary sclerosing cholangitis is thought to be the major hepatobiliary complication of ulcerative colitis."17 A liver biopsy is usually not diagnostic in primary sclerosing cholangitis. ${ }^{1718}$ Concentric periductal fibrosis is considered as more or less pathognomonic for primary sclerosing cholangitis, if other biliary disorders can be excluded but is only found in about one third of the liver biopsies from patients with primary sclerosing cholangitis, ${ }^{18}$ probably because of sporadic distribution. The combination of primary sclerosing cholangitis and ulcerative colitis is found in $50-70 \%$ of all cases with primary sclerosing cholangitis, in particular in patients with involvement of the entire colon. ${ }^{18} 19$ In our study, $7 \%$ of the patients with total colonic involvement showed some periductal fibrosis but none developed any clinical or biochemical evidence of liver disease. The aetiology of periductal fibrosis is not known but repeated episodes of cholangitis, may be one cause. None of the patients had such episodes, however. Based on the present findings we therefore conclude that isolated periductal fibrosis without inflammation seems to be of little clinical significance in patients with ulcerative colitis.

Other reports have shown that cholestasis and bile ductal changes are not related. Several patients have for example, been found to have radiological and histological signs indicating primary sclerosing cholangitis even in the absence of chemical parameters of bile duct disease..$^{20}$ It is also known that asymptomatic patients with primary sclerosing cholangitis may develop symptomatic liver disease several years after the diagnosis has been established..$^{22}$ To our knowledge, however, it is not established whether patients, as those we describe here, without both clinical and biochemical evidence of liver disease with periductal fibrosis, have a 
higher risk to eventually develop a significant liver disease. The three patients we describe have now been observed for more than 20 years and have still not developed any biochemical parameters or symptoms related to liver disease. Accordingly, it seems as if fibrotic changes in liver biopsies from these patients do not indicate a bad prognosis. The exact frequency of primary sclerosing cholangitis as seen with cholangiography among patients with ulcerative colitis is not known. Despite a high frequency of minor histological changes in the liver, only two of our 68 patients developed liver disease symptoms. Six patients were lost at follow up. Four of these may have developed liver disease although it seems unlikely. The two patients, who eventually manifested cirrhosis, had no histological or biochemical signs of liver disease at the time of the first liver biopsy. In the first patient the cause of the cirrhosis is unknown. Even though an endoscopic retrograde cholangiography was not performed, an association with primary sclerosing cholangitis is unlikely, as there were no histological changes typical for primary sclerosing cholangitis and serum alkaline phosphatase activities were normal. Because of frequent blood transfusions non- $A$, non- $B$ hepatitis seems to be a plausible aetiology for the cirrhotic outcome. ${ }^{23}$ The second patient had evidence of an autoimmune liver disease which previously has been described in combination with ulcerative colitis. ${ }^{24}$ The patient finally developed malignant transformation of the bile ducts, a carcinoma known to be associated with ulcerative colitis. ${ }^{25}$ Thus, clear evidence of liver disease associated with ulcerative colitis was only found in one or possibly two (3\%) of our patients.

The range of hepatobiliary complications in patients with ulcerative colitis is reported to be 6 to $25 \% .^{126-28}$ In the present study such complications seen after almost 20 years of follow up were less frequent than might be expected. Accordingly, if there is no clinical or biochemical evidence of hepatobiliary disease during the first years after the onset of ulcerative colitis the patients apparently run a very small risk of developing a hepatobiliary complication.

In conclusion, this study shows that minor histological abnormalities in the liver are common in patients with ulcerative colitis without any clinical or biochemical signs of liver disease. The histological changes are of little help in predicting the risk to develop hepatobiliary complications. The absence of indications of liver disease during the first years after the onset of ulcerative colitis makes it unlikely that the patient will later develop any hepatobiliary com- plications. The present study also suggests that fibrotic changes, especially periductal fibrosis, previously described in patients with primary sclerosing cholangitis ${ }^{15} 18$ do not serve as an unfavourable prognostic marker.

1 Thomas GH. Ulceration of the colon with enlarged fatty liver. Trans Path Soc Phila 1874; 4: 87-8.

2 Kimmelstein $\mathrm{P}$, Large $\mathrm{L}$, Verner $\mathrm{H}$. Liver damage in ulcerative colitis. Am $\mathcal{F}$ Pathol 1952; 28: 259-74.

3 Kleckner M, Stauffner M, Bargen A, Dockerty M. Hepatic lesions in the living patient with chronic colitis as demonstrated by needle biopsy. Gastroenterology 1952; 22: 13-33.

4 De Dombal FT, Goldie W, McK Watts J, Goligher JC Hepatic histological changes in ulcerative colitis. Scand f Gastroenterol 1966; 1: 220-7.

5 Dordal E, Glagov S, Kirsner J. Hepatic lesions in chronic inflammatory bowel disease. Gastroenterology 1966; 52: inflammator

6 Mistilis SP, Skyring AP, Goulston SJ. Pericholangitis and ulcerative colitis. Ann Intern Med 1965; 63: 1-26.

7 Vinnik I, Kern F. Liver diseases in ulcerative colitis. Arch Intern Med 1963; 112: 41-9.

8 Eade MN. Liver disease in ulcerative colitis. Ann Intern Med 1970; 72: 475-87.

9 Warren KW, Athanassides S, Monge JI. Primary sclerosing cholangitis. Am $\mathcal{F}$ Surg 1966; 111: 23-38

10 Thorpe MEC, Scheuer PJ, Sherlock S. Primary sclerosing cholangitis, the biliary tree and ulcerative colitis. Gut 1967 8: $435-48$.

11 Sheperd HA, Selby WS, Chapman RWG, et al. Ulcerative colitis and persistent liver dysfunction. $Q \mathcal{F}$ Med 1983; 52 . 503-13.

12 Schrumpf E, Elgio K, Fausa O, Gjone E, Kolmannskog F Ritland S. Sclerosing cholangitis in ulcerative colitis. Scand Ritland S. Sclerosing cholangitis ir

13 Perrett AD, Higgins G, Johnston H, Massarella GR, Truelove SC, Wright $R$. The liver in ulcerative colitis. $Q \mathcal{F}$ Med 1971; 40: 211-38.

14 Scheuer PJ. Liver biopsy interpretation. London: Bailliere \& Tindall, 1988

15 Nordenvall B, Broström O, Berglund $\mathrm{M}$, et al. Incidence of ulcerative colitis in Stockholm county. Scand $\mathcal{F}$ Gastroenterol 1985; 20: 783-90.

16 Wee A, Ludwig J. Pericholangitis in chronic ulcerative colitis: Primary sclerosing cholangitis of the small bile ducts? Ann Intern Med 1985; 102: 581-7.

17 La Russo NF, Wiesner RH, Ludwig J, Mac Carty RL. Primary sclerosing cholangitis. $N$ Engl f Med 1984; 310 899-903.

18 Chapman RWG, Arborgh BAM, Rhodes JM, et al. Primary sclerosing cholangitis: a review of its clinical features, cholangiography, and hepatic histology. Gut 1980; 21 : 870-7.

19 Wiesner RH, LaRusso NF. Clinicopathologic features of the syndrome of primary sclerosing cholangitis. Gastroenterology 1980; 79: 200-6.

20 Balasubramaniam K, Wiesner RH, LaRusso NF. Primary sclerosing cholangitis with normal serum alkaline phosphatase activity. Gastroenterology 1988; 95: 1395-8.

21 Clements D, Rhodes JM, Elias E. Severe bile duct lesions without biochemical evidence of cholestasis in a case of without biochemical evidence of cholestasis

22 LaRusso NF, Wiesner RH, Ludwig J. Is primary sclerosing cholangitis a bad disease? Gastroenterology 1987; 92: 2031-33.

23 Mattsson L, Weiland O, Glaumann H. Long-term follow-up pf chronic posttransfusion non-A, non-B hepatitis. Liver 1988; 8: $184-8$

24 Olsson R, Hulthén L. Concurrence of ulcerative colitis and chronic active hepatitis. Scand $\mathcal{F}$ Gastroenterol 1975; 10: $331-4$.

25 Mid-Madjlessi SH, Farmer RG, Sivak MV. Bile duct carcinoma in patients with ulcerative colitis. Dig Dis Sci 1987; 32: 145-54.

26 Lupinetti M, Mehigan H, Cameron J. Hepatobiliary complications of ulcerative colitis. Am 7 Surg 1979; 139: 113-8.

27 Dew MJ, Thompson H, Allan RN. The spectrum of hepatic dysfunction in inflammatory bowel disease. $Q \mathcal{F}$ Med 1979 ;
dystic 48: 113-35.

28 Schrumpf E, Fausa O, Kolmannskog F, Elgjo K, Ritland S, Gjone E. Sclerosing cholangitis in ulcerative colitis, a followup study. Scand f Gastroenterol 1982; 17: 33-9. 